\title{
Uso medicinal popular de plantas na comunidade Estreito, zona rural de Redenção do Gurguéia, Piauí, Brasil
}

Popular medicinal use of plants in the Estreito rural community of Redenção do Gurguéia, Piauí, Brazil

Uso medicinal popular de las plantas en la comunidad rural Estreito de Redenção do Gurguéia, Piauí, Brasil

\author{
Anaiane Guimarães Nunes da Silva \\ ORCID: https://orcid.org/0000-0001-7792-3098 \\ Universidade Federal do Piauí, Brasil \\ E-mail: salvadoranaiane@gmail.com \\ Thalita Brenda dos Santos Vieira \\ ORCID: https://orcid.org/0000-0002-6171-8644 \\ Universidade Estadual do Piauí, Brasil \\ E-mail: thalytabrenda1998@gmail.com \\ Antonia Amanda Cardoso de Almeida \\ ORCID: https://orcid.org/0000-0003-3250-0336 \\ Universidade Federal do Piauí, Brasil \\ E-mail: antoniaamanda.almeida@gmail.com \\ Rusbene Bruno Fonseca de Carvalho \\ ORCID: https://orcid.org/0000-0002-5993-1729 \\ Universidade Federal do Piauí, Brasil \\ E-mail: rusbenecarvalho@gmail.com
}

\begin{abstract}
Resumo
A evidencias do uso das plantas medicinais vem desde os primórdios da humanidade, pois utilizavam destas para curar/tratar pessoas de suas comunidades. Plantas medicinais tem por definição como todo vegetal que possui, em um ou mais órgãos, constituintes químicos que podem ser utilizados como agentes terapêuticos, bem como materiais de partida para a síntese de drogas. Neste contexto, esta pesquisa teve como objetivo investigar o saber popular sobre as plantas medicinais utilizadas para a promoção da saúde e cura de enfermidades na comunidade de Estreito, povoado que fica localizado a dezoito quilômetros da cidade de Redenção do Gurguéia. Incialmente, foi realizado um estudo de caracterização da área de estudo buscando o reconhecimento da área e uma aproximação com a população residente. Em seguida, realizou-se entrevistas com questões subjetivas e por fim, na terceira etapa envolveu o registro fotográfico do material botânico utilizando-se do método de "trilha livre". Neste estudo verificou-se um considerável número de espécies vegetais utilizadas com fins medicinais. O conhecimento dos antepassados é algo que evidencia os possíveis efeitos benéficos de tratamento/cura das plantas medicinais na comunidade Estreito.
\end{abstract}

Palavras-chave: Plantas medicinais; Promoção da saúde; Uso terapêutico.

\begin{abstract}
Evidence of the use of medicinal plants comes since the beginning of humanity, as they used these plants to cure or treat people in their communities. Medicinal plants are defined as plants that have, in one or more organs, chemical constituents that can be used as therapeutic agents, as well as materials for the synthesis of drugs. This research aimed to investigate the popular knowledge on medicinal plants used to health promotion and cure illnesses in the community of Estreito, which is located eighteen kilometers from the city of Redenção do Gurguéia. A characterization study of the study area was carried out, seeking recognition of the area and an approximation with the resident population. Then, there were interviews with subjective questions and, finally, the third stage involved the photographic record of the botanical material using the free trail method. In this study a substantial number of species used for medicinal purposes was verified. Knowledge of ancestors is something that highlights the beneficial healing possible effects of medicinal plants in the Estreito community.
\end{abstract}

Keywords: Medicinal plants; Health promotion; Therapeutic use.

\section{Resumen}

La evidencia del uso de plantas medicinales proviene de los albores de la humanidad, ya que las usaban para curar / tratar a las personas en sus comunidades. Las plantas medicinales se definen como toda planta que tiene, en uno o más 
órganos, constituyentes químicos que se pueden utilizar como agentes terapéuticos, así como materiales de partida para la síntesis de fármacos. En este contexto, esta investigación tuvo como objetivo investigar el conocimiento popular sobre las plantas medicinales utilizadas para promover la salud y curar enfermedades en la comunidad de Estreito, una aldea ubicada a dieciocho kilómetros de la ciudad de Redenção do Gurguéia. Inicialmente se realizó un estudio de caracterización del área de estudio, buscando el reconocimiento del área y una aproximación con la población residente. Luego, se realizaron entrevistas con preguntas subjetivas y, finalmente, la tercera etapa involucró el registro fotográfico del material botánico mediante el método de rastro libre. En este estudio se verificó un número considerable de especies vegetales utilizadas con fines medicinales. El conocimiento de los antepasados es algo que resalta los posibles efectos beneficiosos del tratamiento / curación de plantas medicinales en la comunidad de Estreito.

Palabras clave: Plantas medicinales; Promoción de la salud; Uso terapéutico.

\section{Introdução}

A evidência do uso das plantas medicinais vem desde os primórdios da humanidade, pois utilizavam destas para curar/tratar pessoas de suas comunidades. $\mathrm{O}$ uso de plantas para enfermidades é evidenciado até os dias de hoje, muitas vezes, por falta de recursos financeiros. Tal conhecimento pode ser passado por gerações (Mattos et al., 2018).

As plantas medicinais são definidas como toda e qualquer planta que possui substâncias com propriedades terapêuticas, seja em partes específicas ou como todo, que é utilizada pelo homem com propósitos de cura (Brasil, 2010). A Organização Mundial da Saúde (OMS), reconhece a importância das plantas medicinais para a promoção da saúde, bem como incentiva o resgate, o reconhecimento e a valorização das práticas tradicionais e populares de uso de plantas e remédios caseiros (OMS, 2013). A organização ainda ressalta a importância da Medicina Tradicional para atenção primária a saúde, uma vez que $80 \%$ da população utiliza práticas tradicionais nos seus cuidados básicos de saúde, sendo $85 \%$ plantas ou preparações destas (Brasil, 2016).

Segundo Almeida (2015), a OMS também incentiva o uso das plantas medicinais em países onde o custo dos medicamentos é muito alto e são ricos em biodiversidade, como é o caso do Brasil. Em concordância, Cavaglier (2014) afirma que,

(...) mesmo com o avanço da medicina em diversas partes do mundo, no Brasil, as plantas medicinais costumam ser uma das alternativas para parte da população, principalmente a de baixa renda, devido a diversos fatores, dentre os quais, o custo alto dos medicamentos industrializados e o acesso restrito a um sistema de saúde de qualidade. Em contrapartida, o uso deste tipo de terapia tem crescido também entre as pessoas de maior poder aquisitivo, na busca por opções terapêuticas mais saudáveis (Cavaglier, 2014, p. 56).

No Brasil, a utilização de plantas com fins medicinais tem suas bases na prática indígena, que com o passar dos anos foi influenciada pelos mais diferentes grupos étnicos, esses trouxeram valiosas contribuições para o desenvolvimento da pesquisa em produtos naturais (Viegas Junior et al., 2006). O conhecimento tradicional indígena pode ser encontrado em todo o território brasileiro (Alves et al., 2015; Rocha; Marisco, 2016). Outra cultura que trouxe bastante conhecimento sobre plantas medicinais, foi a africana, os escravos trouxeram na sua bagagem um conhecimento vasto sobre este tema (Silva et al., 2019).

Além desses conhecimentos adquiridos através dos primeiros habitantes que povoaram o Brasil, o conhecimento tradicional passado de pai para filho, foi fundamental para esse relacionamento das plantas com o homem. A medicina popular é orientada por muitos conhecimentos acumulados diante da relação direta dos seus membros com o meio ambiente e da propagação de experiências transmitido por diferentes gerações, tornando comum a sobrevivência de parte desse conhecimento tradicional (Moreira et al., 2002).

As comunidades rurais destacam-se como fontes riquíssimas de conhecimento, com um acervo de informações incalculável. Couto-Waltrich et al. (2017) afirmam que comunidades rurais têm naturalmente proximidade com a biodiversidade vegetal no local onde vivem. Sales et al. (2015) destacam o Brasil como sendo a maior diversidade vegetal do 
planeta e ampla sociodiversidade, com enorme potencial no desenvolvimento da fitoterapia. Nesse contexto, a prática do uso é muito comum nas zonas rurais e essa forma de utilizar as plantas como medicamento é algo característico principalmente das comunidades de baixa renda (Teixeira \& Melo, 2006; Neto, 2014; Santos et al., 2016; Silva et al., 2019).

É valido ressaltar que nos dias atuais mesmo muitos possuindo acessos aos medicamentos que podem ser adquiridos em farmácias, muitas comunidades mantém o hábito de preparo de tratamentos caseiros à base de plantas para controle de enfermidades (Coan \& Matias, 2014). Entretanto, é valido ressaltar que a falsa ideia que a planta medicinal é segura não quer dizer que as plantas não podem ser prejudiciais, elas produzem substâncias químicas capazes de interferir na saúde de forma positiva ou negativa, essa informação é conhecida desde tempos dos nossos antepassados. Assim, existe a necessidade de atrelar o conhecimento empírico com o conhecimento cientifico para assim expandir ainda mais os efeitos benéficos das plantas medicinais (Bieski, 2020).

Nesse contexto, levando em consideração que existe um grande acervo de plantas medicinais na comunidade Estreito, povoado que fica localizado próximo a cidade de Redenção do Gurguéia no Piauí, e que os moradores costumam muitas vezes substituir o medicamento farmacológico adquiridos em farmácias pelo o uso natural de plantas para tratar doenças corriqueiras como: gripes, diarreias, inflamação, entre outros, surgiu o interesse em estudar a temática deste trabalho. Assim, o objetivo do presente trabalho é investigar o saber popular sobre as plantas medicinais utilizadas para a promoção da saúde e cura de enfermidades na comunidade de Estreito da cidade de Redenção do Gurguéia no Piauí, Brasil.

\section{Metodologia}

\subsection{Caracterização da área de estudo}

A zona rural Estreito é um povoado que fica localizado a dezoito quilômetros da cidade de Redenção do Gurguéia (Figura 1), apresentam temperaturas mínimas de $20{ }^{\circ} \mathrm{C}$ e máximas de $32{ }^{\circ} \mathrm{C}$, com clima quente e semiúmido.

Figura 1 - Localização da área de estudo.
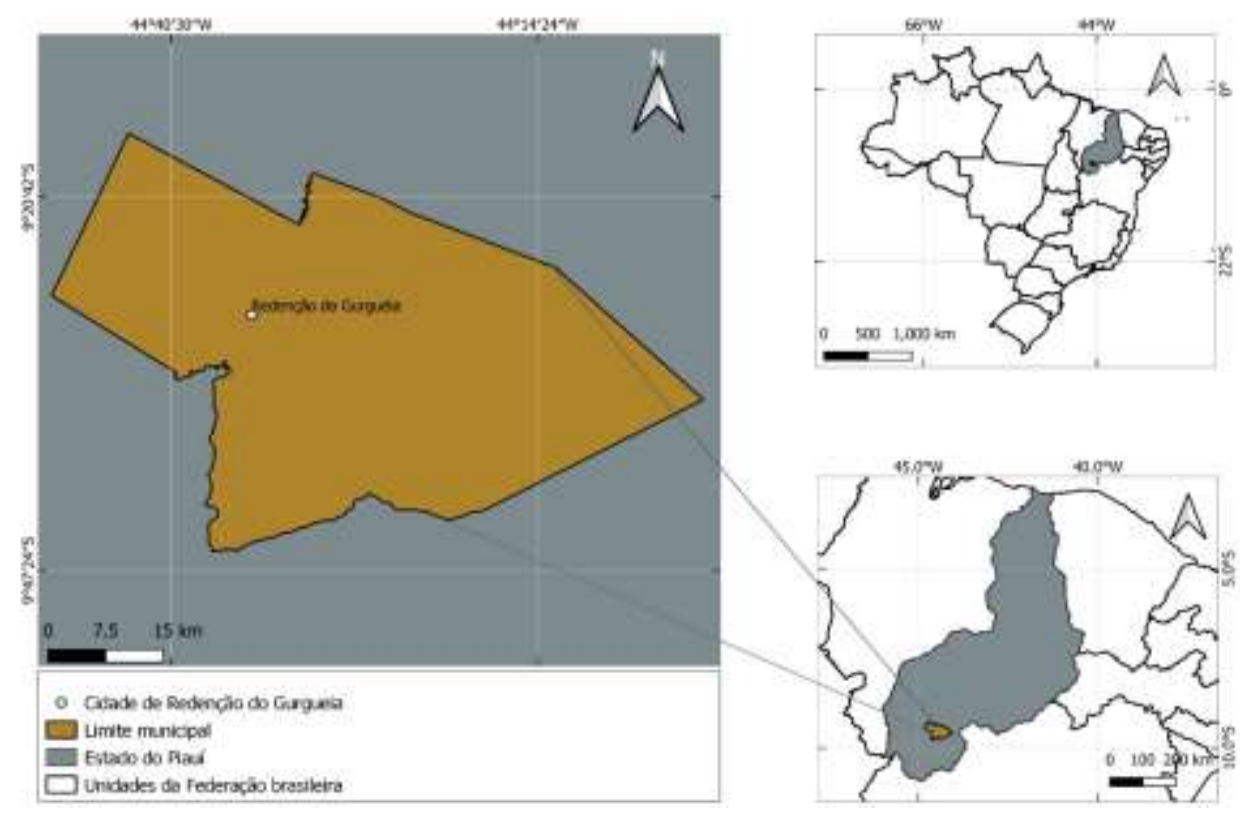

Fonte: Autores (2021).

De acordo com dados coletados na Prefeitura Municipal de Redenção do Gurguéia, o povoado Estreito possui dez famílias residentes que vivem em casas de adobo, recobertas com reboco, com energia elétrica e água encanada, sem 
esgotamento sanitário, algumas dessas famílias possui acesso a telefone rural e acesso internet por torres de sinal. Na comunidade tem um posto de saúde, onde há atendimentos semanalmente, segundo o secretário de saúde na Unidade Básica de Saúde (UBS) do Estreito há consultas médico, odontológico, de enfermagem, dispensação de medicamentos, atendimento a gestantes, crianças, diabéticos, hipertensos.

Sobre a infraestrutura, de acordo com secretário da cidade foram realizadas a recuperação da estrada vicinal que dá acesso à comunidade do Estreito, reforma do posto de saúde em parceria com a secretaria de saúde, tudo para melhoria de vida da comunidade. Nesse sentido, uma característica importante e que tornou o local como área de estudo foi baseada em prévias observações, por meio das quais foi possível identificar a presença de um grande acervo de espécies frutíferas, alimentares, condimentares e também medicinais observadas tanto nos quintais, como nas roças e lugares próximos a comunidade.

\subsection{Coleta e análise dos dados}

O trabalho de campo foi realizado no mês de maio do ano de 2021. Iniciou-se-com visitas de reconhecimento da área, buscando uma breve aproximação com a população residente, para reconhecimento sociocultural da comunidade e a relação destes com plantas utilizadas popularmente com fins medicinais.

Em uma segunda etapa do trabalho realizou-se entrevistas com questões subjetivas (Tabela 1), cujo critério para escolha dos participantes considerou a disponibilidade dos participantes em responder as perguntas, bem como o tempo de residência no povoado e a idade, entendendo que os mais idosos e os que moram há mais tempo na comunidade sejam os que possuem maior conhecimento sobre o uso destas plantas medicinais (plantas utilizadas, doses, modo de preparo, indicações terapêuticas, preferência pelo uso em relação a outros tratamentos).

A terceira etapa envolveu o registro fotográfico do material botânico, utilizando-se do método de "trilha livre" onde os entrevistados guiaram os pesquisadores num caminho por eles estabelecido mostrando as plantas utilizadas para fins medicinais (Albuquerque et al., 2008). Por fim, a partir de cada espécie registrada, foram realizadas pesquisas em bases de dados nacionais objetivando a identificação das plantas e se suas respectivas propriedades medicinais já são descritas na literatura.

Tabela 1 - Perguntas abordadas nas entrevistas de campo.

\begin{tabular}{|c|c|c|}
\hline $\mathbf{N}^{\mathbf{0}}$ & Perguntas & Pontos abordados \\
\hline 1 & Qual é a sua idade? & \multirow{4}{*}{ Perfil dos entrevistados } \\
\hline 2 & Qual é o seu nível escolar? & \\
\hline 3 & Qual é a sua ocupação? & \\
\hline 4 & A quanto tempo você reside na comunidade? & \\
\hline 5 & Você já fez uso de alguma planta para uso medicinal? & \multirow[b]{2}{*}{ Identificação de uso } \\
\hline 6 & $\begin{array}{l}\text { Quais as plantas existentes no seu quintal ou nas proximidades é utilizada } \\
\text { para fins medicinais? Quais são elas? }\end{array}$ & \\
\hline 7 & $\begin{array}{l}\text { Quais doenças são tratadas com as respectivas plantas? (Ex: Febre, gripe, } \\
\text { dor, diarreia, etc.). }\end{array}$ & Enfermidades \\
\hline 8 & Quais as partes utilizadas? (Ex: Folhas, casca, raízes). & \multirow[b]{2}{*}{ Uso das plantas medicinais } \\
\hline 9 & $\begin{array}{l}\text { Qual a frequência de uso destas plantas? (Ex. Todo dia, raramente, se } \\
\text { preciso). }\end{array}$ & \\
\hline 10 & $\begin{array}{l}\text { Como chegou ao seu conhecimento que tal planta pode ser utilizadas para } \\
\text { fins medicinais? Quem lhe indicou? }\end{array}$ & Conhecimento popular \\
\hline
\end{tabular}

Fonte: Autores (2021).

É valido ressaltar que devido a pandemia foram tomadas todas medidas de segurança para proteção dos entrevistados, levando em consideração as recomendações da OMS. 


\section{Resultados e Discussão}

\subsection{Perfil dos entrevistados}

$\mathrm{Na}$ comunidade Estreito existe dez famílias residentes, dentre os entrevistados observou-se que cerca de $75 \%$ destes fazem parte do sexo feminino, e $25 \%$ do sexo masculino, notando-se assim que a mulher neste processo se encontra na vanguarda por estar inteiramente ligada à família e é detentora de determinados conhecimentos e habilidades cognitivas nativas nesta área do saber, ajudando a aliviar o sofrimento de famílias (Kramer, 2010).

A mulher por estar mais em casa, por se dedicar a família e aos afazeres domésticos tem o domínio em pratica e uso das plantas medicinais, notando que a mesma busca nos efeitos curandeiros das plantas o alivio da dor dos seus familiares. Em concordância, Pulga (2013), afirma que o conhecimento sobre plantas medicinais está intimamente ligado ao papel das mulheres dentro da família. Para Albuquerque et al. (2012) é importante a consideração do conhecimento da mulher nas várias esferas da medicina local na busca de medicamentos através de plantas medicinais.

Em relação a idade cerca de $75 \%$ tem entre 50 e 70 anos, o que destaca o conhecimento vindo de gerações passadas. Em consonância, Zeni et al. (2017) ressalta que a idade dos moradores é bastante significativa na sua pesquisa com plantas medicinais por representar o conhecimento passado de geração em geração. Nesse contexto, tal conhecimento é evidenciado nesta pesquisa e a idade torna-se um fator positivo para obter e catalogar as plantas existentes na comunidade.

Outro fator que também é determinado pela idade diante desta pesquisa de campo é o tempo de residência na comunidade, notando que certa de $75 \%$ dos moradores residem na comunidade a mais de 20 anos e somente $12,5 \%$ moram nela com menos de 5 anos. Tal fato mostra que o tempo traz consigo os conhecimentos e bagagens que são passadas por seus pais, avós, tios, já que as maiorias estão residindo na comunidade porque herdaram um pedaço de terra de seus pais e assim cuidam e cultivam plantas naquele lugar.

Com relação ao nível de escolaridade cerca $62,5 \%$ tem apenas o ensino fundamental incompleto, $12,5 \%$ tem o ensino fundamental completo, $12,5 \%$ tem o ensino médio completo e $12,5 \%$ tem o ensino superior completo. Esses resultados evidenciam um índice de alto de analfabetismo, e este baixo nível de escolaridade muitas vezes é o que impede os moradores de buscar esclarecimentos junto aos médicos e faz com que esses acabam recorrendo ao uso das plantas medicinais.

Entretanto, para Pires et al. (2014), o nível de escolaridade se mostra independente quando se trata do uso de plantas medicinais, podendo afirmar que o uso de plantas medicinais como opção de tratamento terapêutico ocorre de forma semelhante em pessoas com níveis menores de escolaridade e pessoas com maiores níveis de escolaridade. Assim, acredita-se que a falta de escolaridade da maioria não impede que os mesmos tenham um conhecimento e passem esse conhecimento para outros, pois esses conhecimentos foram adquiridos através da oralidade que é passado pelas gerações passadas.

\subsection{Identificação de uso, enfermidades e uso das plantas medicinais}

Os moradores entrevistados fazem o uso das plantas medicinais diariamente como prevenção de doenças corriqueiras, tais como: diarreias, gripe, entre outros. Ver-se a vivacidade desse fato quando ao chegar para realizar a entrevista, um dos moradores estava consumido o sumo do mastruz para tratar infecções, sendo que o mesmo conta que faz uso diariamente dessa e de outras plantas medicinais.

Esses dados corroboram com o apresentado por Teixeira et al. (2006), que constatou em uma pesquisa realizada no Estado de Pernambuco que $100 \%$ dos entrevistados têm em casa pelo menos uma espécie de planta medicinal, adotada no tratamento de alguma enfermidade. Nesse sentido, foi elaborado uma tabela com as plantas citadas por cada morador participante, bem como os nomes populares e científicos, partes utilizadas e as enfermidades que serviam, constatando a presença de quarenta e duas plantas medicinais (Tabela 2). O registro fotográfico (material suplementar) das plantas utilizadas 
pela comunidade pode ser visualizada por meio do link https://drive.google.com/file/d/1M6pu2MJ9Amk04b_VLE88edQVtkiZL8X/view?usp=sharing.

Tabela 2 - Nomes populares e cientifico, partes utilizadas e doenças tratadas das plantas medicinais.

\begin{tabular}{|c|c|c|c|}
\hline $\begin{array}{c}\text { Nome popular adotado } \\
\text { na comunidade }\end{array}$ & Nome científico & Parte utilizada & Uso pela comunidade \\
\hline Casaca & Não encontrado & Casca & $\begin{array}{c}\text { Comida ensacada no } \\
\text { estômago, para construção } \\
\text { dos alimentos. }\end{array}$ \\
\hline Cajá & Spondias mombin & Casca & $\begin{array}{l}\text { Desinflamação, sara } \\
\text { ferimentos. }\end{array}$ \\
\hline Angico & Anadenanthera colubrina & Casca & Gripe e pneumonia \\
\hline Malva de porco & Phyllanthus niruri & Raiz e folha & Diarreia \\
\hline Alho & Allium sativum & Raiz & Gripe, pressão alta \\
\hline Folha de carne & Casearia Sylvestris $S w$ & Folha & Falta de apetite \\
\hline Pau rato & Caesalpiniaceae & Folha e a casca & Diarreia \\
\hline Pau ferro & Caesalpinia ferrea & Fruto e a casca & Diabetes e gripe \\
\hline Cebola & Allium cepa & Raiz & Gripe \\
\hline Coentro & Coriandrum sativum & Sementes & Gripe \\
\hline Casca de laranja & Citrus sinensis & Casca & Diarreia \\
\hline Limão & Citrus limon & Frutos & Gripe \\
\hline Babosa & Aloe vera & Miolo & Gripe, vermes \\
\hline Acerola & Malpighia glabra Linné & Frutos e folhas & Gripe \\
\hline Folha de algodão & Gossypium spp & Folhas & Gripe \\
\hline Mastruz & $\begin{array}{l}\text { Chenopodium } \\
\text { ambrosioides }\end{array}$ & Folhas & Infecção \\
\hline Anador & Alternanthera brasiliana & Folhas & Gripe \\
\hline Semente de imburana & Commiphora leptophloeos & Sementes & Diarreia \\
\hline Folha de cana & Sacchaeum officinarum & Folhas & Hipertensão \\
\hline Urucum & Bixa orellana & Folhas e sementes & Diabetes \\
\hline Casca de ameixa & Ximenia americana & Cascas & Inflamação \\
\hline Aroeira & Schinus terebinthifolia & Cascas & Inflamação \\
\hline Canela de velho & Miconia albicans & Cascas & Dor de coluna \\
\hline Erva cidreira & Melissa officinalis & Folhas & Calmante \\
\hline Gengibre & Zingiber officinale & Raízes & Gripe \\
\hline Romã & Punica granatum & Cascas e sementes & $\begin{array}{c}\text { Inflamação de garganta, } \\
\text { gripe }\end{array}$ \\
\hline Folha santa & Maytenus ilicifolia & Folhas & Inflamação \\
\hline Hortelã & Mentha spicata & Folhas & Gripe e gastrite \\
\hline Boldo & Peumus boldus & Folhas & Desintoxicação de fígado \\
\hline Manjericão & Ocimum basilicum & Folhas & Dor de cabeça, gripe \\
\hline Malvão & Plectranthus amboinicus & Folhas & Inflamação, gases e gripe \\
\hline Açafrão & Curcuma longa & Raízes & $\begin{array}{l}\text { Inflamação de garganta, } \\
\text { dor de cabeça e gripe }\end{array}$ \\
\hline Olho de goiaba & Psidium guajava & Frutos em formação & Diarreia \\
\hline Folha de pitomba & Talisia esculenta & Folhas & $\begin{array}{l}\text { Aumentar dor na hora do } \\
\text { parto }\end{array}$ \\
\hline Carrapicho mulher dama & Acanthospermum australe & Toda a planta & Gripe \\
\hline Tamarindo & Tamarindus & Frutos & Ressecamento \\
\hline $\begin{array}{l}\text { Folha amarela do mamão e } \\
\text { cacho do mamão macho }\end{array}$ & Carica papaya & Folhas e cachos & $\begin{array}{l}\text { Labirintite, antidepressivo, } \\
\text { congestão e náuseas }\end{array}$ \\
\hline Folhas de ata & Annona squamosa & Folhas & Anemia \\
\hline Coronha & Vachellia farnesiana & Sementes e cascas & Epilepsia, dor no pulso. \\
\hline Bucheira & Luffa aegyptiaca & Folhas & Diabetes \\
\hline Maracujá do mato & Passiflora cincinnata & Frutos & Hipertensão \\
\hline Pião roxo & Jatropha gossypiifolia & Folhas & Cicatriza ferimentos \\
\hline
\end{tabular}

Fonte: Autores (2021). 
Com relação as enfermidades detectaram-se 15 tipos diferentes de doenças tratadas pelas plantas, nota-se que a maioria delas são utilizadas para enfermidades comuns como gripes (12), infecções (9), diarreias (5), hipertensão (3), diabetes (3), totalizando certa de 76,19\%, conforme observado na Figura 2.

Figura 2 - Doenças tratadas pelas plantas pela comunidade.

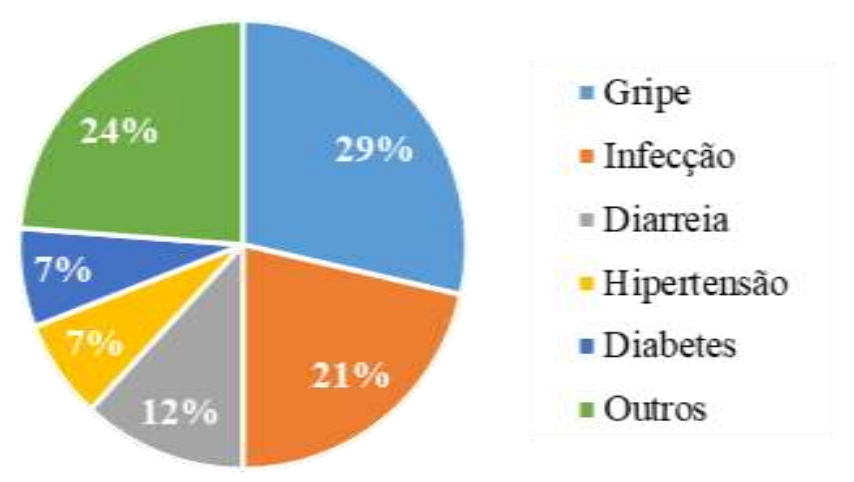

Legenda: O termo "Outros" inclui: cicatrizante, anemia, epilepsia, labirintite antidepressivo, congestão e náuseas, ressecamento, aumentar dor de parto, inflamação/ infecção, desintoxicação, calmante, falta de apetite, comida ensacada no estômago. Fonte: Autores (2021).

Nesse contexto, Ghilardi (2020) afirma que o uso dos remédios caseiros oriundo da flora local pode trazer desenvolvimento sustentável, uma vez que a medicina popular e a cura das doenças mais comuns se tornam acessíveis à população, e junto, vem o cuidado com a natureza, com a cultura e com os conhecimentos ancestrais. Ainda de acordo com o autor, as plantas mais utilizadas dependem da estrutura de cada comunidade, das necessidades ligadas à cultura, ambiente e enfermidades mais comuns.

O uso diário destas plantas medicinais, em alguns casos é de 2 a 3 vezes ao dia. Observou-se por meio do convite dos participantes que nas geladeiras dos entrevistados havia sempre algum tipo de chá, garrafada e/ou melado feitos para uso cotidiano, pois segundo eles na maioria das vezes opta-se primeiro pelo uso destes. Um exemplo que pode ser citado, é quando se tem uma diarreia, faz-se primeiro a utilização de um chá e de acordo com os moradores na maioria das vezes se tem um resultado positivo, não havendo a necessidade de uso de medicamentos farmacológicos.

As plantas medicinais podem ser consideradas fonte alternativa de tratamento para diversas doenças, principalmente em comunidades mais carentes, entretanto é valido ressaltar que as orientações dos profissionais de saúde são necessárias para evitar riscos no agravo da saúde, de maneira eficaz e segura (Paixão et al., 2016).

O saber popular pode ser utilizado como base para a pesquisa científica sobre plantas medicinais, contribuindo com os profissionais da área da saúde no sentido de buscar subsídios para a introdução de espécies em programas de fitoterapia na rede de atendimento básico, além de contribuir para o sistema local de saúde e ajudar a desenvolver o potencial econômico inerente às plantas de valor medicinal (Negrelle et al., 2007).

Ainda foi possível observar que a parte mais utilizada das plantas foram as folhas com cerca de 38,09\%, seguido pela a casca $(16,6 \%)$, a raiz $(9,52 \%)$, o fruto $(7,14 \%)$, semente $(4,76 \%)$ e o miolo da planta $(2,38 \%)$. A planta em sua totalidade teve seu uso em 2,38\% e em 19,04\% usam duas partes combinadas, tais como: frutos e as folhas, folhas e as casca, raízes e as folhas, frutos e as cascas, cascas e as sementes, folhas e o cachos, e folhas e a sementes.

As folhas são as partes mais citadas em estudos sobre uso medicinal, o que está atrelado a sua disponibilidade na planta durante a maior parte do ano, e por apresentarem princípios ativos e terapêuticos que curam enfermidades comuns na 
população brasileira (Guerra et al., 2012; Silva et al., 2015). O uso das folhas implica na conservação do recurso vegetal, por não impedir a reprodução e o desenvolvimento vegetativo e, principalmente, não degradar o sistema reprodutivo da planta (Pilla et al., 2006).

Uma das plantas que foi bastante citada pelos moradores, Boldo (Peumus boldus), usada para desintoxicação do fígado, também foi uma das mais utilizadas por moradores da cidade de Floriano no Piauí em estudo realizado por Vasconcelos, Alcoforado e Lima (2010), sendo a folha a parte da planta mais utilizada pela população na preparação de chás.

A hortelã (Mentha spicata) foi citada por mais de 50\% dos moradores como sendo uma planta que combate a gripe. Em um outro estudo realizado por Adjuto (2008), comprovou que a hortelã possui atividade antiparasitária, na eliminação de amebas e giárdias. Liporacci e Simão (2013) também encontraram a hortelã como a mais citada em levantamento realizado em bairro mineiro do município de Ituiutaba, no entanto 50\% dos usuários a indicavam para doenças do sistema respiratório, salientando a importância do etnoconhecimento local e regional sobre o uso das plantas medicinais.

Outra planta que segundo os moradores combate à gripe é a babosa (Aloe vera) e o gengibre (Zingiber officinale). Coutinho et al. (2002) em seus estudos diz que a Babosa possui ação antiparasitária. Já o gengibre além de propriedades terapêuticas, é de uso corrente na culinária, como condimento (Camargo, 2006; Beal, 2006). Outro estudo afirma que os extratos do gengibre têm sido utilizados por muitos séculos, pela medicina popular para curar e tratar diversas patologias (Rogrigues; Lira, 2013).

\subsection{Conhecimento popular}

Observou-se que $100 \%$ dos entrevistados atribuíram o conhecimento as gerações passadas, afirmaram que aprenderam com seus avós e pais, passando assim de uma geração para outra, notando-se que este é o um fator importante essa relação de aprendizado. Esse conhecimento é vindo desde a existência do homem, e esse vai transferindo por gerações.

A tradição familiar é a razão pela qual os indivíduos mais utilizam as plantas medicinais, pois os pais passam para seus filhos os costumes e ensinamentos (Siqueira \& Pereira, 2014). Manter vivo o conhecimento das gerações passadas é fundamental para a continuação do saber a respeito destas, pois essas experiências trazem vivacidade para a cultura local.

Diante disto Martins et al. (2005), afirma que a melhor opção é manter o respeito pela cultura, tradição e usos e costumes de um povo, pois cada povo numa comunidade tem suas particularidades e possui informações muito valiosas no tratamento de enfermidades com plantas medicinais. Em relação a oralidade, o contato familiar evidencia a importância da família na transmissão do conhecimento através da oralidade (Motta et al., 2016).

O Brasil tem uma rica história de uso das plantas medicinais no tratamento dos problemas de saúde da população, uso este construído com base na experiência e transmitido de forma oral (Bruning et al., 2012). A utilização de plantas com fins medicinais no Brasil tem suas bases na prática indígena, esses trouxeram importantes contribuições para o desenvolvimento da pesquisa em produtos naturais, do conhecimento da relação entre a estrutura química de um determinado composto e suas propriedades biológicas (Santos, 2000; Viegas Junior et al., 2006).

\section{Conclusão}

Neste estudo verificou-se um considerável número de espécies vegetais utilizadas com fins medicinais. O conhecimento dos antepassados é algo que evidencia os efeitos benéficos de cura das plantas medicinais da comunidade Estreito, ficou evidente um saber que abrange grande quantidade de plantas que curam e tratam doenças corriqueiras, essa transmissão é notável apesar da maioria ter pouco estudo, o que não impede que esses propaguem o conhecimento sobre essas. Durante todo processo nota-se que esses fazem uso das plantas medicinais como primeira opção, e caso não resolva, então procuram medicamentos farmacológicos, através de consultas medicas. Isso porque de acordo com eles os efeitos colaterais 
dessas são menos agravantes para o organismo. Assim ficou evidente que é necessário a preservação desse conhecimento e também das plantas existentes nessa comunidade.

Dentro deste contexto, este foi o primeiro trabalho realizado na comunidade Estreito zona rural de Redenção do Gurguéia, Piauí, Brasil, e busca refletir a riqueza do conhecimento popular aliado ao tratamento de doenças na medicina tradicional associada ao uso de plantas medicinais, além de estimular pesquisas visando o surgimento de trabalhos futuros, sejam no aspecto etnobotânico e/ou farmacológico. É valido ressaltar que todo o resultado obtido nesta pesquisa será disponibilizado para a comunidade em forma de panfleto com os acervos das plantas medicinais encontradas, com fotos ilustrando cada planta, bem como suas respectivas atividades farmacológicas/medicinais já públicas em acervo científico.

\section{Agradecimentos}

A todos os participantes da pesquisa, pela receptividade, disponibilidade e paciência em responder aos questionamentos e por nos guiar na realização dos registros fotográficos.

\section{Referências}

Adjuto, E. N. P. (2008). Caracterização morfológica e de óleo essencial de seis acesos de Hortelanzinho (Mentha spp.) (Dissertação de Mestrado). Faculdade de Agronomia e Medicina Veterinaria, Universidade de Brasilia, Brasilia, DF, Brasil.

Albuquerque, U. P., Lucena, R. F. P. \& Cunha, L. V. F. C. C. (2008). Métodos e técnicas na pesquisa etnobotânica. Editora Comunigraf/ NUPEEA: Recife, Brasil.

Albuquerque, U. P. \& Medeiros, P. M. (2012). Revisões sistemáticas e metanálises aplicadas à pesquisa etnobiológica. Revista Etnobiologia e Conservação.

Almeida, I. A. \& Sousa, R. T. O. (2015). Benefício das Plantas Medicinais na utilização pelos Professores em Uma Escola Pública. Volume I. Universidade Estadual Londrina - Paraná, 5p.

Alves, J. J. P., Lima, C. C. de., Santos, D. B., Bezerra, P. D. F. (2015). Conhecimento popular sobre plantas medicinais e o cuidado da saúde primária: um estudo de caso da comunidade rural de mendes, São José de Mipibu/RN. Carpe Diem: Revista Cultural e Científica do Unifacex, $13(1)$, 136-156.

Beal, B. H. (2006). Atividade antioxidante e identificação dos ácidos fenólicos do gengibre (Zingiber officinale Roscoe) 2006.87 p. Dissertação (Mestrado em Ciência dos Alimentos) - Centro de Ciências Agrárias, Universidade Federal de Santa Catarina.

Bieski, I. G. C. (2020). Jardim Medicinal no seu Quintal: Saberes e Fazeres para o uso dos chás e conhecimentos das plantas medicinais nos quintais. Juína.

Brasil. (2016). Ministério da Saúde. Secretaria de Ciência, Tecnologia e Insumos Estratégicos. Departamento de Assistência Farmacêutica. Política e Programa Nacional de Plantas Medicinais e Fitoterápicos/Ministério da Saúde, Secretaria de Ciência, Tecnologia e Insumos Estratégicos, Departamento de Assistência Farmacêutica - Brasília: Saúde. http:/bvsms.saude.gov.br/bvs/publicacoes/politica_programa_nacional_plantas_medicinais_fitoterapicos.pdf.

Brasil. (2010). $R D C n^{o} 10$ de março de 2010. Dispõe sobre a fitoterapia de drogas vegetais junto à Agência Nacional de Vigilância Sanitária, de 10 de março de 2010 .

Bruning, M. C. R., Mosegui, G. B. G. \& Viana, C. M. M. (2012). A utilização da fitoterapia e de plantas medicinais em unidades básicas de saúde nos municípios de Cascavel e Foz do Iguaçu-Paraná: a visão dos profissionais de saúde. Ciência e Saúde coletiva, 17(10), 2.675-2.685.

Camargo, L. C. S. (2006). Efeito antiinflamatorio do extrato de Zingiber officinale aplicado por fonoforese sobre o edema de pata de ratos. 89 p. Dissertação (Mestrado em Ciências Biológicas) - Instituto de Pesquisa e Desenvolvimento, Universidade do Vale do Paraíba, São José dos Campos.

Carneiro, V. P. P., Gumy, M. P., Otânio, J. K., Menetrier, J. V., Medeiros, K. A., Bonkoski, V. R., Gasparotto junior, A., Estevan, D. A., Castro, T. E., Lourenço, E. L. B., Velasquez, L. G. \& Jacomassi, E. (2020). Perfil do uso de plantas medicinais por moradores da área rural de um Município do Estado do Paraná. Research, Society and Development, 9(10), 1-27.

Carvalho, R. B. F. (2017). Desenvolvimento tecnológico de um complexo de inclusão do nerolidol com ciclodextrina e avaliação de propriedades farmacológicas. 178p. Tese (Pós-graduação em Biotecnologia, Rede Nordeste de Biotecnologia - RENORBIO) - Universidade Federal do Piauí, Teresina, Piauí.

Cavaglier, M. C. S. \& Messeder, J. C. (2014). Plantas Medicinais no Ensino de Química e Biologia: Propostas Interdisciplinares na Educação de Jovens e Adultos. Revista Brasileira de Pesquisa em Educação em Ciências, 14(1), 55-71.

Coan, C. M. \& Matias, T. (2014). A utilização das plantas medicinais pela comunidade indígena de Ventarra Alta-RS. Sábios: Revista Saúde e Biologia, 9(1) 11-19.

Coutinho, D. F. Coutinho, D. F., Travassos, L. M. A. \& Amaral, F. M. M. (2002). Estudo etnobotânico de plantas medicinais utilizadas em comunidades indígenas no estado do maranhão- Brasil. Visão Acadêmica, 3(1), 7-12. 
Couto-Waltrich, C., Boff. P. \& Boff, M. I. C. (2017). Etnoconhecimento associado às amoreiras-brancas (Rubus spp.) ocorrentes na floresta ombrófila mista, Santa Catarina, Brasil. Revista de la Faculdade de Agronomia, La Plata, 116(1), 13-18.

Ghilardi, T. (2020). Relação do uso de plantas medicinais na produção de remédios caseiros com a sustentabilidade: uma revisão. Escola Nacional de Administração Pública, Brasília-DF.

Guerra, N. M., Ribeiro, J. E. S., Carvalho, T. K. N., Pedrosa, K. M., Felix, L. P. \& Lucena, R. F. (2012). Usos locais de espécies vegetais nativas em uma comunidade rural no semiárido nordestino (São Mamede, Paraíba, Brasil). Revista de Biologia e Farmácia, $184-210$.

Hoeffel, J. L. M., Gonçalves, N. M., Fadini, A. A. B.\& Seixas, S. R. C. (2011). Conhecimento tradicional e uso de plantas medicinais nas APAS'S Cantareira/SP e Fernão Dias/MG. Revista VITAS - Visões Transdisciplinares sobre Ambiente e Sociedade. n.1.

Kramer, K. L. (2010). Cooperative breeding and its significance to the demographic success of humans. Annual Review of Anthropology, (39), 417-436.

Liporacci, H. S. N. \& Simão, D. G. (2013). Levantamento etnobotânico de plantas medicinais nos quintais do Bairro Novo Horizonte, Ituiutaba, MG. Revista Brasileira de Plantas Medicinais, 15(4), 529-540.

Martins, A. G., Rosário, D. L., Barros, M. N. \& Jardim, M. A. G. (2005). Levantamento etnobotânico de plantas medicinais, alimentares e toxicas da Ilha do Combu, Município de Belém, Estado do Pará. Revista Brasileira de Farmacognosia, (86), 31-40.

Martins, M. C., \& Garlet, T. M. B. (2016). Desenvolvendo e divulgando o conhecimento sobre plantas medicinais. Revista Eletrônica em Gestão, Educação e Tecnologia Ambiental, 20(1), 438-448.

Mattos, G., Camargo, A., De Sousa, C. A. \& Zeni, A. C. L. B. (2018). Medicinal plants and herbal medicines in primar y health care: The perception of the professionals. Ciência e Saúde Coletiva, 23(11), 3735-3744.

Moreira, R. C. T. et al. (2002). Abordagem Etnobotânica acerca do Uso de Plantas Medicinais na Vila Cachoeira, Ilhéus, Bahia, Brasil. Acta Farmcéutica Bonaerense, 21(3), 205-211.

Motta A. O. \& Lima D. C. S. Vale C. R. (2016). Levantamento do uso de plantas medicinais em um centro de educação infantil em Goiânia-Go. Revista da Universidade Vale do Rio Verde, 14(1): 629-646.

Negrelle, R. R. B. \& Fornazzari, K. R. C. (2007). Estudo etnobotânico em duas comunidades rurais (Limeira e Ribeirão Grande) de Guaratuba (Paraná, Brasil). Revista Brasileira de Plantas Medicinais, 9(2), 36-54.

Neto, F. R. G. (2014). Estudo Etnobotânico de plantas medicinais utilizadas pela Comunidade do Sisal no município de Catu, Bahia, Brasil. Revista Brasileira de Plantas Medicinais, (16), 856-865.

OMS, Organizacion Mundial de La Salud. (2013). Estrategia de la sobre medicina tradicional 2014-2023. Ginebra, Editora Organización Mundial de la Salud, 75p.

Paixão, J. Á. \& Conceição, R. S., Santana Neto, A. F., Neto, J. F. A., Santos, U. S. (2016). Levantamento bibliográfico de plantas medicinais comercializadas em feiras da Bahia e suas interações medicamentosas. Revista Eletrônica de Farmácia, 13(2), 71-81.

Pereira, A. R. A., Velho, A. P. M., Cortez, D. A. G., Szerwieski, L. L. D. \& Cortez, L. E. R. (2016). Uso tradicional de plantas medicinais por idosos. Revista Rene, 17(3), 427-434.

Pilla, M. A. C., Amorozo, M. C. L. \& Furlan, A. (2006). Obtenção e uso das plantas medicinais no distrito de Martim Francisco, Municipio de Mogi-Mirim, SP, Brasil. Acta Botânica Brasilica, 20(4), 789-802.

Pires, I. F. B., Souza, A. A., Feitosa, M. H. A. \& Costa, S. M. (2014). Plantas medicinais como opção terapêutica em comunidades de Montes Claros, Minas Gerais, Brasil. Revista Brasileira Plantas Medicinais, 16(2), 426-433.

Pulga, V. L. (2013). Contribuições do movimento de mulheres camponesas para a formação em saúde. Trabalho, educação e saúde, 11(3), 573-590.

Rocha, R. \& Marisco, G. (2016). Estudos etnobotânicos em comunidades indígenas no Brasil. Revista Fitos, 10(2), $95-219$.

Sales, M. D. C., Sartor, E. B. \& Gentilli, R. M. L. (2015). Etnobotânica e Etnofarmacologia: medicina tradicional e bioprospecção de fitoterápicos. Revista Salus, 1(1), 17-26.

Santos, A. B. N. et al. (2016). Plantas medicinais conhecidas na zona urbana de Cajueiro da Praia, Piauí, Nordeste do Brasil. Revista Brasileira Plantas Medicinais, (18), 442-50.

Santos, F. S. D. (2000). Tradições populares de uso de plantas medicinais na Amazônia. História, Ciências, Saúde-Manguinhos, vol. VI, p. 919-939.

Silva, A. B. et al. (2019). Extrato etanólico das folhas de Raphanus sativus L. var. oleifera Metzg (nabo forrageiro): efeitos anti-hiperglicêmico, antidislipidêmico e antioxidante em ratos com Diabetes Mellitus tipo 1. Revista Fitos, (13), 38-48.

Silva, P. H., Barros, M. S., Oliveira, Y. R. \& Abreu, M. C. (2015). A Etnobotânica e as plantas medicinais sob a perspectiva da valorização do conhecimento tradicional e da conservação ambiental. Revista de Ciências Ambientais, 9(2), 67.

Siqueira, A. B. \& Pereira, S. M. (2014). Abordagem etnobotânica no ensino de Biologia. REMEA-Revista Eletrônica do Mestrado em Educação Ambiental, $31(2), 247-260$

Teixeira, S. A. \& Melo, J. I. M. (2006). Plantas medicinais utilizadas no município de Jupi, Pernambuco, Brasil. Iheringia, Série Botânica, (6), 15-11. 
Research, Society and Development, v. 10, n. 17, e27101724350, 2021

(CC BY 4.0) | ISSN 2525-3409 | DOI: http://dx.doi.org/10.33448/rsd-v10i17.24350

Vasconcelos, D. A., Alcoforado, G. G. \& Lima, M. M. O. (2010). Plantas medicinais de uso caseiro: conhecimento popular da região do centro do município de Floriano/PI. Congresso de Pesquisa e Inovação da Rede Norte Nordeste de Educação Tecnológica, Maceió, Alagoas, Brasil, n. 5.

Viegas Junior, C., Bolzani, V. S. \& Barreiro, E. J. (2006). Os produtos naturais e a química medicinal moderna. Química Nova, 29(2), $326-337$.

Zeni, A. L. B., Parisotto, A. V., Mattos, G. \& Santa Helena, E. T. (2017). Utilização de plantas medicinais como remédio caseiro na Atenção Primária em Blumenau, Santa Catarina, Brasil. Revista Ciência \& Saúde Coletiva, 22(8), 2703-2712. 